\title{
FÍSICA BÁSICA: INCIDENCIA DE LA INSTRUCCIÓN SOBRE LOS ERRORES CONCEPTUALES
}

COLOMBO de CUDMANI, L. y CUDMANI, C. Instituto de Fisica. Universidad Nacional de Tucumán.

\section{SUMMARY}

The incidence of some instructional aspects on the persistency of preconcepts and errors has been analized. Confusions, derived from both, the no-integration of friction forces into the introductory experiencies to the mechanic laws study and the fact of not taking into account quantitative criterions to control the laws and models validity, are particulary studied.

\section{INTRODUCCIÓN}

En los últimos años se ha analizado en diversos trabajos (Viennot, 1979; Clement, 1982; Solís Villa, 1984; Boido, 1985; Nussbaum, 1981) cl problema de los «preconceptos» o "errores conceptuales» en el aprendizaje de los principios de la Mecánica; en particular el de las concepciones no-newtonianas que establecen la proporcionalidad entre la fuerza y la velocidad ( $f=k . v$ ) en contraposición con la correspondiente relación entre la fuerza y aceleración $(F=m . a)$. En estos artículos se han analizado aspectos históricos, psicológicos, lógi$\cos$, instruccionales y se ha puesto de manifiesto su persistencia aún después de haber recibido la instrucción académica correspondiente (Abeledo, 1985; Peters, 1982; Trowbridge y otros, 1980-1981).

Nos proponemos analizar en este trabajo, basándonos en observaciones derivadas de la experiencia docente, algunos aspectos de la enseffanza cuya incidencia en esta persistencia y resistencia a la instrucción podrian ser importantes.

\section{OBSERVACIONES INTRODUCTORIAS}

Consideraremos, a modo de introducción en la problemática que nos ocupa, respuestas de los estudiantes a una pregunta evaluativa, que nos impresionó vivamente: en un trabajo práctico sobre el concepto de medición y sus errores se pidió a los alumnos que explicitaran cuáles eran los supuestos necesarios para la validez de la relación entre fuerza y aceleración $(F=m . a)$. Se trataba de una revision sobre el tema con 120 estudiantes de $2^{\circ}$ año de Licenciatura en Física e Ingenie- ría Eléctrica que ya habian aprobado la unidad «Mecánica».

Encontramos sorprendentes respuestas:

«La fuerza debe ser constante a fin de que la masa fuera constantes.

«La fuerza debe ser mayor que la de interacción para que el cuerpo se muevan.

«La fuerza de roce debe ser constante para que F sea constante».

Pero más allá de estas confusiones accidentales, la respuesta que nos interesó particularmente fue que:

«Para que valga dicha relación la fuerza de roce debe ser cero (o despreciable»).

El $44 \%$ de los alumnos se adhirieron a esta proposición.

A fin de indagar con mayor precisión este foco de confusión, y evitar posibles falsas interpretaciones derivadas de no tener claro qué se entiende por «supuesto para la validez de una leys, se incluyo, en una prueba de las que se toman habitualmente, el siguiente item de evaluación:

«Indique cuál/es de la/s siguiente/s proposición/es son correctas y explique por qué:

«Para que pueda aplicarse la relación de Newton $\mathrm{F}=\mathrm{m} . \mathrm{a}$, deben cumplirse las siguientes condiciones:

a) $\mathrm{F}$ debe ser constante

b) in debe ser la masa inercial

c) las fuerzas de roce deben ser despreciables

d) F debe ser la fuerza resultante». 
El muestreo se realizó con 350 alumnos de $2^{\circ}$ de Ingeniería y se repitió con otro grupo similar en el siguiente periodo lectivo. Los resultados fueron significativamente similares entre sí y al obtenido en la primera encuesta: entre el 42 y el $45 \%$ de los estudiantes, consideraron ucorrectas a la afirmación c).

Este resultado apunta en la misma dirección que otras numerosas observaciones que muestran que el rozamiento no está debidamente integrado en la instrucción lo cual dificuita la comprensión y aplicación de las leyes de la Mecánica.

En los trabajos de laboratorio se observa, por otra parte que, cuando el comportamiento de un sistema físico se aparta del previsto, por regla general se atribuye la divergencia al efecto de las fuerzas de rozamiento, muchas veces frente a situaciones en las que esta hipótesis no resiste el análisis más superficial.

Veamos un ejemplo concreto que aclarará esta idea:

Ejemplo - El roce en su forma de «régimen turbulento"s es también el causante de todos los comportamientos anómalos de los fluidos. Cuando los alumnos analizan los métodos para medir viscosidad de un aceite de máquina, descartan el método de Hagen-Pouseilie, de salida por un tubo capilar, lo cual es correcto, pero dan como razón que, "puesto que la viscosidad es muy grande, el régimen es turbulento" por lo cual no se puede aplicar el método aludido.

En este ejemplo, la confusión se detectó en el $94 \%$ de los cursos. Las muestras fueron de más de 500 estudiantes, a lo largo de cuatro perfodos lectivos.

Estas y muchas otras observaciones muestran que el efecto de las fuerzas de roce no está correctamente elaborado. La señal «apartamiento del valor teórico esperado» desencadena en forma automática, irreflexiva (Piaget, 1973; Cudmani y otros, 1981), la respuesta «la causa es el roce».

\section{LAS FUERZAS DE ROCE EN LA INSTRUCCIÓN}

Una investigación sobre el tipo de ejemplos fenomenológicos en los cuales se introdujo el concepto de masa en la instrucción recibida por estos alumnos muestra que se usó la experiencia de Mach, mediciones sobre un riel de aire, u otro tipo de ejemplos cuyo común denominador fue considerar el roce despreciable.

La revisión de los textos de mayor uso entre los estudiantes mostró también que, en la mayoría de los casos, las experiencias introductorias suponian despreciables las fuerzas de roce.

Desde el punto de vista lógico para la construcción sistemática de la disciplina, parece razonable comenzar el estudio de un problema usando supuestos simplificatorios que hagan más sencillo su análisis.
Pero lo simple suele ser también lo más abstracto y por ende lo más alejado de la experiencia previa y del pensamiento intuitivo que, como señala Ausubel (Ausubel y otros, 1978), debe ser usado como eficaz anclaje (subsunsores) para construir el pensamiento lógico y sistemático en un esquema igualmente eficiente para interpretar la realidad.

Si se quiere orientar la enseftanza de las ciencias, estableciendo un paralelismo con «el proceso de producción científica", será necesario tener muy en cuenta no sólo la estructura conceptual de la disciplina, sino también la estructura cognoscitiva del alumno; «la importancia de los paradigmas conceptuales como origen y término de una investigación, tiene su equivalente en la enseñanza en la importancia de la estructura cognoscitiva del alumno" (Gil Pérez, 1983).

Como se analiza en numerosos trabajos sobre el modelo constructivista para el aprendizaje de las ciencias, resulta de fundamental importancia generar situaciones que permitan al estudiante un cambio de paradigma, (Khum, 1973; Driver y Oldham, 1986).

Para ello es necesario modificar los arraigados preconceptos que se han estructurado, a través de múttiples experiencias y observaciones cotidianas, en una coherente, aunque contradictoria e intuitiva, interpretación de la realidad: la sepistemología del sentido común o metodología de la superficialidad" (Carrascosa y Gil, 1985).

Pero para poder lograr ese desarraigo consideramos que es necesario partir de esas mismas experiencias y observaciones en lugar de substituirlas por otras que, con sus artificios simplificatorios, no resultan convincentes para el alumno.

Introducir los conceptos de la mecánica con ejemplos y experiencias en las cuales las situaciones concretas se han simplificado de modo que sea la realidad la que se adecúa al modelo, significa, a nuestro entender, una práctica opuesta al fundamento epistemológico de una ciencia fáctica donde son los modelos los que, en sucesivas aproximaciones, deben adecuarse a la realidad.

Se produce así, en el alumno, una actitud dual: hay una física cuyas leyes se aplican en clases, laboratorios, respuestas de examen, situaciones académicas en general pero que no funciona frente a los fenomenos de la realidad cotidiana.

No es raro pues, que, desde el punto de vista psicológico, el alumno incurra en confusiones como las observadas; la condición «roce igual a cero» impuesta para simplificar el análisis de una situación se convierte en un supuesto necesario para la validez de la ley fundamental.

A fin de aclarar mejor esta idea que consideramos válida no sólo para la mecánica sino también para otros temas de la física, se introducirán algunas precisiones respecto al concepto de ley física. 


\section{LAS LEYES FÍSICAS Y SU RELACIÓN CON LOS FENOMMENOS}

Bunge $(1963,1973)$ destaca en su análisis del concepto de ley científica la diferencia entre un enunciado nomológico o fórmula legaliforme (pauta invariante a nivel lógico, que designa como ley II) y una ley nomopragmática (pauta invariante a nivel pragmático o ley IIr). Las del primer tipo son proposiciones conceptuales, «constructos» (Bunge, 1981) que «tienen como referente inmediato" a un modelo teórico y no pueden ser comprobadas directamente por la experiencia. Este es cl caso de las que habitualmente llamamos «las leyes de la Físican tales como $\mathrm{F}=\mathrm{m} . \mathrm{a}$.

El segundo tipo, fórmula nomopragmática, se refiere por lo menos parcialmente a la experiencia y contiene datos e información fácticas tales como condiciones iniciales, constantes características de un sistema, etc. Un ejemplo sería: «si se lanza un cuerpo verticalmente hacia arriba ambos con velocidad inicial $v_{0}$ la altura máxima $h$ que alcanza será igual $a h=1 / 2 v_{0}^{2} / g . »$

Los ejemplos particulares que se usan para introducir el concepto de masa son casos particulares de este segundo tipo de leyes y es para ellas que se hace el supuesto (fuerza de roce igual a cero). Pero igualmente se hubieran podido tomar ejemplos donde este supuesto no fuera necesario. De este modo se comprende que el estudiante extienda el supuesto que fue necesario en todos los ejemplos analizados a la correspondiente ley en el nivel lógico, $F=$ m.a.

De allí a aceptar que, cuando el supuesto no se cumple, es la velocidad la que resulta de la aplicación de una fuerza, hay un sólo paso. Si se partiera de ejemplos que muestren «anomalias» (Posner 1982) en las cuales las ideas intuitivas fracasan, en lugar de «fabricar» ejemplos ajenos a la experiencia del alumno, es posible que se obtuvieran mejores resultados.

Esta discusión parece apuntar a la necesidad de destacar durante la instrucción los limites de validez y los supuestos que debe satisfacer una situación real para que a ella pueda aplicarse un modelo teórico, para el cual vale la ley Il correspondiente. Creemos que no se insiste suficientemente en este tipo de consideraciones durante la instrucción.

Relacionado con esta necesidad surge, a nuestro criterio, otro aspecto que debiera ser revalorizado y debidamente integrado. Es el análisis del papel que juegan los crrores de medición en la instrucción.

\section{EXPLICITACIÓN Y ANÁLISIS DE SUPUESTOS}

Habitualmente, y tal como ocurre con las fuerzas de roce, los errores de medición se consideran en la instrucción como un tema o capitulo especial dedicado a su estudio. Luego se dejan de lado; y no se intcgran al análisis de los otros temas que se estudian.

Sin embargo, si consideramos que para poder describir la realidad con las leyes y modelos de la disciplina es imprescindible definir con exactitud en qué medida ellos son adecuados para la situación problemática a la que se aplican, es decir, en qué medida se cumplen los supuestos y los límites de validez a los que aludimos anteriormente, no cabe duda que el único criterio válido es el que surge de considerar los errores en la medición de las magnitudes que intervienen.

Por regla general, este análisis se trata superficialmente o no se considera en absoluto, no sólo en el caso del rozamiento sino en muchas otras situaciones. Cuando se imparte información o se plantean ejemplos de ejer citación en clase, en textos, en trabajos prácticos, se da por sentado que los supuestos se cumplen y se elude el problema mediante consideraciones tales como «supongamos el roce despreciable», "considere que la masa es puntual», "considere el campo lejos de los bordes», "suponga un solenoide muy largo". Ante un caso concreto ¿cómo puede el estudiante decidir por sí mismo si en efecto puede considerar con exactitud que se dan estas condiciones presupuestas? Para hacerlo será menester usar como referencia el error de medición.

No hay dudas de que el análisis de cstas cuestiones aumentará la complejidad del problema, pero si no se instruye al estudiante sobre los criterios que le permitirán dar las respuestas necesarias, se abre cada vez más la brecha entre la física y los fenomenos (Halbwachs, 1985) o se la transforma en una aproximación cualita* tiva o inexacta de la realidad.

En el apéndice se plantean algunos problemas de cjercitación y ejemplificación que a nuestro criterio pue. den mejorar la comprensión de cómo deben aplicarse las conceptuaciones a las situaciones reales.

Este enfoque es útil también para contrarrestar la tendencia, común entre los estudiantes, a reducir la ley física a su expresión matemática simbólica. Se despoja a la relación fáctica de todo su ropaje de signifi. cado no explícito en la formulación matemática, de todo el contexto verbal que la complementa (Padrón, 1982).

De este modo, no sólo se dejan de lado supuestos y límites de validez sino que se termina perdiendo el significado físico de los símbolos mismos (Padrón, 1982).

Veamos un ejemplo muy demostrativo de esta situación: de 150 estudiantes de $2^{\circ}$ curso de Ingeniería Eléctrica a los que se interrogó en un itern de evaluación sobre "qué propiedades debe tener la superficie de Gauss, en el teorema que vincula el campo F de una carga $q$ mediante la relación: $\varepsilon_{0} / \overrightarrow{\mathrm{E}}$. ds $=\mathrm{a}$

el $20 \%$ respondió que «debia ser de material aislante» y $; 40 \%$ ! «que debia ser conductora». 


\section{CONCLUSIONES}

El análisis efectuado en este trabajo parece sugerir dos líneas orientadoras para mejorar los resultados en el aprendizaje de conceptos y leyes.

Por un lado, debería incorporarse un análisis profundo y detallado del rol que juegan las fuerzas de roce en las experiencias sobre mecánica. Las experiencias introductorias en particular, deberían elegirse de modo tal que no siempre sea necesario suponer $F_{k}=0$. Esto puede ser útil para ayudar a desarraigar los preconceptos respecto a proporcionalidad entre fuerza y velocidad. Además se podrían evitar las confusiones que lievan a que se atribuyan a las fuerzas de roce todas las discrepancias entre modelo y realidad.

\section{REFERENCIAS BIBLIOGRÁFICAS}

ABELEDO y otros, 1985, Dificultades conceptuales en un problema de Fisica elemental. Memorias de REF XV, pág. 119. (Edit. Univ. Nac. de Tucumán).

AUSUBEL, D.P., NOVAK, J.D., HANESIAN, H., 1978, Educational Psychology: a cognitive view, N.Y. Hoit Rinehart and Weston.

BOIDO, G., 1985, Concepciones prenewtonianas en el aprendizaje de la Física, Memorias de REFIV, pág. 119, (Edit. Univ. Nac. de Tucumán).

BUNGE, M., 1963, La ciencia, su método y su filosofia. (Siglo XX. Buenos Aires.

BUNGE, M., 1981, Epistemologia. (Ed. Ariel, Barcelona).

BUNGE, M., 1973, La investigación cientifica, pág. $375 \mathrm{y}$ sub. (Ed. Ariel, Barcelona).

CARRASCOSA y GIL, 1985, La metodología de la superficialidad y el aprendizaje de las ciencias, Enseñanza de las Ciencias, vol. 3 (2), pág. 110-120.

CLEMENT, J., 1982, Student's preconceptions in introductory mechanics, Am. J. Physics, 50(1), pág. 66 y sub.

CUDMANI, L., LEWIN, A.M., LOZANO, S., 1981, El problema de aplicación como ejercicio de aprendizaje operativo, Rev. Brasilera de Física, vol. 11, n 1, pág. 269 y sub.

DRIVER, R. y OLDHAM, V., 1986, A constructivist aproach to curriculum development in science, Studies in Sc. Educ, 13, pág. 116-122.

GIL PÉREZ, D., 1983, Tres paradigmas básicos en la enseñanza de las ciencias, Enseñanza de las Ciencias, vol. 1, pág. 29.
Por otro lado, surge el valor de integrar la instrucción los criterios que derivan del análisis de errores de medición para controlar la validez de supuestos y límites de validez de un determinado modelo cuando se aplican las leyes que de él derivan (leyes nomológicas) a una situación fáctica mediante una ley nomopragmá. tica, (Bunge, 1973).

En estas propuestas cuando se habla de instrucción sc entiende no sólo las técnicas de transmisión de información (conferencias, clases, textos, etc.) sino también trabajos de ejercitación y laboratorio.

Se están implementando estas ideas en cursos básicos universitarios y secundarios a fin de poder tener resultados de una experiencia controlada. Las conclusiones serán objeto de otro trabajo.

HALBWACHS, F, 1985, La Física del profesor entre la Fisica del físico y la Fisica del alumno, Articulo reproducido en Revista de enseñanza de la Física, A.P.F.A., vol. $1, n^{\circ} 2$, pág. 87 .

KUHN, T.S. 1971, La estructura de las resoluciones cientificas, (Fondo de Cultura económica, México).

NUSSBAUM, J., 1981, Towards, the diagnosis by science teachers of pupils' Misconceptions: an Exercise with students Teachers, Eur. J. Sci. Educat., vol. 3, p. 159-169.

PADRON, H., 1982, La pérdida del logos. Razón discursiva y razón calculadora, Seminario de Epistemologia de las ciencias. (UNSTA, Tucumán).

PETERS, P.C., 1982, Even honors students have conceptual difficulties with physics, Am. J. Phys. 50(6), pág. 501 y sub.

PIAGET, J,, 1973, Psicología de la inteligencia, pág. 122 y sub. (Edit. Psique, Buenos Aires).

POSNER, G. y colaboradores, 1982, Accomodation of a scientific conception: Toward a theory of conceptual change, Sci. Educ. 66 (2), pág. 211 .

SOLIS VILLA, R., 1984, Ideas intuitivas y aprendizaje de las ciencias, Enseñanza de las Ciencias, vol. 2, $\mathrm{n}^{\circ} 2$, pag. 83 y sub.

TROWBRIDGE, D. MCDERMOTT, L., 1980, Investigation of student understanding of the concept of velocity in one dimensione, Am. J. Phys. 48(12) p. 1020 y sub.

VIENNOT, L., 1979, Spontancous reasoning in elementary dinamics, Eur. J. Sci. Educ., vol. 1, pág. 205 y sub. 


\section{APÉNDICE}

1) Un bloque de $0,21 \mathrm{~kg}$. se desliza sobre un plano que forma un ángulo de $21,5^{\circ}$ con la horizontal.

Cuando se calcula la aceleración del bloque ¿dentro de qué orden de precisión se puede considerar despreciable la fuerza de roce?

a) si la superficie no está lubricada $(\mathrm{u}=0,5)$

b) si la superficie está lubricada $(u=0,05)$

2) Un estudiante se propone determinar $g$ (aceleración de la gravedad) midiendo el período de un péndulo construido con un alambre de acero de aproximadamente $50 \mathrm{~cm}$ de longitud y $2 \mathrm{~mm}^{2}$ de sección del cual se suspende una esfera del mismo material que pesa 1 $\mathrm{kg}$.

A. Qué error introduce en la medición si considera: a) a la esfera como una masa puntual

b) al hilo como inextensible.

B. En una experiencia piloto se comprueba que el mayor error de medicion se comete en la determinación del periodo y es del orden del $2 \%$. ¿Puede, sin error apreciable, hacer las simplificaciones enunciadas en a) y b)?

3) Se quiere medir la viscosidad $\eta$ de un líquido con precisión del $5 \%$. El orden de magnitud de $\eta$ es 10 poise y el de la densidad de $1 \mathrm{gr} / \mathrm{cm}^{3}$. El método elegido consiste en medir la velocidad de régimen de municiones de acero que se dejan caer en un tubo con el liquido.

Bajo estas condiciones ise puede despreciar:

a) las fuerzas de roce? ¿por qué?

b) el empuje que actúa sobre la munición? ¿por qué? 